\title{
TESTE DE TETRAZÓLIO EM SEMENTES DE LEUCENA ${ }^{1}$
}

\author{
CAROLINE JÁCOME COSTA², CAROLINA PAULA DOS SANTOS ${ }^{3}$
}

\begin{abstract}
RESUMO - O teste de tetrazólio constitui alternativa promissora para avaliação da viabilidade e vigor de sementes. Além de possibilitar a obtenção de resultados rápidos, gera informações que permitem a identificação das causas de perda da viabilidade das sementes, sendo recomendado para avaliação da viabilidade de sementes que necessitam ser semeadas logo após a colheita e para complementar os resultados do teste de germinação de sementes que apresentam dormência. A eficiência do teste depende do desenvolvimento de metodologia adequada para cada espécie, que recomende condições apropriadas de pré-condicionamento, preparo e coloração das sementes. Objetivou-se, com o trabalho, avaliar métodos destinados ao preparo e coloração de sementes de leucena para o teste de tetrazólio e verificar sua eficiência para avaliar a viabilidade das sementes. Para os experimentos, foram utilizadas sementes da cultivar 'Cunningham' e do híbrido interespecífico (Leucaena leucocephala $\mathrm{x}$ L. diversifolia) CPAC 4111. Duas metodologias destinadas ao preparo das sementes para a coloração foram testadas: corte lateral e corte lateral seguido de imersão das sementes em água, a $30{ }^{\circ} \mathrm{C}$, por uma hora, e posterior remoção do tegumento. Para a coloração, foram avaliadas as concentrações de $0,075 \%, 0,15 \%$ e $1 \%$ da solução de tetrazólio e os períodos de duas e quatro horas, a $35^{\circ} \mathrm{C}$. Os resultados obtidos foram comparados com os resultados dos testes de germinação e emergência de plântulas. $\mathrm{O}$ teste de tetrazólio é eficiente para avaliar a viabilidade de sementes de leucena, por meio do corte lateral seguido da remoção do tegumento e imersão em solução de tetrazólio a $0,15 \%$, por duas horas.
\end{abstract}

Termos para indexação: Leucaena leucocephala, Leucaena diversifolia, germinação, emergência, viabilidade.

\section{TETRAZOLIUM TEST IN LEUCAENA SEEDS}

ABSTRACT - The tetrazolium test is a useful alternative for seed viability and vigor evaluation because besides producing rapid results it can also identify the causes of lost viability. This test is recommended for the evaluation of the viability of seeds that have to be sown soon after harvest and also complements germination tests for seeds that present dormancy. For each species, the efficiency of the tetrazolium test depends on the development of appropriate methodology that includes appropriate methods for pre-conditioning, preparation and staining of the seeds. We evaluated preparation and staining methods for applying the tetrazolium test to leucaena seeds and verified the efficiency of this test for assessing the viability of leucaena seeds using seeds from Leucaena leucocephala cv. Cunningham and the interspecific hybrid (L. leucocephala $\mathrm{x}$ diversifolia CPAC 4111. Two seed preparation methods were tested, a lateral cut and a lateral cut followed by immersion in water for one hour at $30{ }^{\circ} \mathrm{C}$ and subsequent seed coat removal. The seeds were stained with $0.075 \%, 0.15 \%$ or $1 \%$ tetrazolium solution for two or four hours at 35

\footnotetext{
${ }^{1}$ Submetido em 01/10/2009. Aceito para publicação em 08/12/2009.

${ }^{2}$ Eng. Agr., Pesquisadora da Embrapa Cerrados. BR 020, Km 18, Rodovia Brasília-Fortaleza, CEP 73310-970 Planaltina, DF. E-mail: caroline.costa@, cpac.embrapa.br.
}

${ }^{3}$ Estudante de Biologia, Estagiária da Embrapa Cerrados. E-mail: carolina. paula@cpac.embrapa.br. 


\begin{abstract}
${ }^{\circ} \mathrm{C}$ and then evaluated. The tetrazolium test results were compared with the results of standard germination and seedling emergence tests. Our results show that subjecting the seeds to a lateral cut and seed coat removal and then submersing them in $0.15 \%$ tetrazolium solution for two hours is an efficient method for evaluating leucaena seed viability.
\end{abstract}

Index terms: germination, emergency, Leucaena diversifolia, Leucaena leucocephala, viability.

\section{INTRODUÇ̃̃O}

A leucena (Leucaena leucocephala (Lam.) de Wit) é uma espécie pertencente à família Fabaceae, originária da América Central. Denominada a "alfafa dos trópicos", é considerada uma espécie versátil, de elevado valor nutricional, com múltiplos usos. Graças ao sistema radicular profundo, resiste à seca e constitui opção para a suplementação animal durante a estiagem (Souza e Espíndola, 2000; Manella et al., 2002). Para a formação de novas áreas de pastagens que incluam a leucena, a qualidade das sementes é fundamental, ressaltando a importância do desenvolvimento e aprimoramento de testes que permitam a avaliação da qualidade das sementes.

$\mathrm{O}$ teste de germinação tem sido o método tradicionalmente empregado para avaliação da viabilidade das sementes. Todavia, em alguns casos, a obtenção dos resultados requer longos períodos, limitando a tomada de decisão durante o controle de qualidade das sementes. No caso de sementes de leucena, por exemplo, os resultados do teste de germinação são obtidos após dez dias (Brasil, 1992). Por essa razão, busca-se desenvolver e aperfeiçoar testes rápidos e confiáveis que ofereçam a caracterização do potencial fisiológico das sementes.

Dentre esses testes, destaca-se o teste de tetrazólio, que consiste em um método rápido para avaliar a viabilidade e o vigor de sementes, baseando-se na atividade de enzimas do grupo das desidrogenases, presentes nos tecidos vivos. Neste teste, as sementes permanecem em contato com uma solução incolor de cloreto de tetrazólio (2,3,5 trifenil cloreto de tetrazólio), que é absorvida pelos tecidos da semente. Nos tecidos vivos que apresentam atividades respiratória e metabólica normais, as enzimas do grupo das desidrogenases liberam íons hidrogênio $\left(\mathrm{H}^{+}\right)$com os quais o sal 2,3,5 trifenil cloreto de tetrazólio reage, formando um composto insolúvel e estável, de coloração avermelhada, denominado trifenilformazan (Delouche et al., 1976). A intensidade e localização das partes coloridas e descoloridas são utilizadas para a interpretação do teste. As sementes viáveis tendem a absorver a solução de tetrazólio lentamente, desenvolvendo coloração mais suave do que sementes deterioradas, que adquirem coloração rosa forte. Os tecidos mortos, nos quais não há atividade dessas enzimas, são caracterizados pela coloração branca ou amarelados e textura flácida (França Neto, 1999). O teste de tetrazólio permite rápida avaliação da viabilidade e do vigor das sementes e, por vezes, auxilia na identificação das causas da perda da viabilidade das mesmas, além de requerer equipamentos simples e de baixo custo para sua execução (Delouche et al., 1976; França Neto et al., 1998; França Neto, 1999).

Para que os resultados do teste sejam satisfatórios, é necessário que a solução de tetrazólio seja adequadamente absorvida pelas sementes. Por essa razão, algumas espécies necessitam passar por etapas preparatórias previamente à imersão na solução de tetrazólio. Em geral, as sementes devem ser pré-embebidas em água antes de serem submetidas à imersão na solução de tetrazólio, com o objetivo de ativar o metabolismo enzimático, uma etapa conhecida como précondicionamento. Após o pré-condicionamento, muitas espécies ainda necessitam de técnicas preparatórias, que envolvem, frequentemente, a punção, o corte e/ou a remoção do tegumento. No caso das sementes de leucena, que apresentam tegumento impermeável, são recomendados vários métodos para o preparo das sementes antes da coloração: a punção, o corte ou lixamento dos tegumentos, em zona não decisiva, oposta ao eixo embrionário, na parte distal da semente; corte longitudinal através dos tegumentos ou remoção da parte distal da semente. Para que ocorra a coloração, recomendase que as sementes sejam submetidas às concentrações de 0,5 a 1,0\% da solução de tetrazólio, por 6 a 24 horas (Brasil, 1992). Verifica-se que as recomendações das Regras para Análise de Sementes são inespecíficas quanto à concentração da solução de tetrazólio a ser empregada, além de requerer longos períodos para a obtenção dos resultados. Esses fatores reforçam a necessidade de aperfeiçoar as recomendações para realização do teste de tetrazólio em sementes de leucena. Objetivou-se, com o presente trabalho, avaliar métodos destinados ao preparo e coloração das sementes de leucena para o teste de tetrazólio e avaliar sua eficiência.

\section{MATERIAL E MÉTODOS}

O trabalho foi conduzido no Laboratório de Análise 
de Sementes da Embrapa Cerrados, em Planaltina-DF. Empregaram-se sementes da cv. 'Cunningham' e do híbrido interespecífico L. leucocephala x L. diversifolia, CPAC 4111. Previamente à execução dos experimentos, as sementes foram escarificadas manualmente, lixando-se a extremidade oposta ao eixo embrionário, até a exposição dos cotilédones.

Para avaliação de metodologias destinadas ao preparo e coloração para o teste de tetrazólio, sementes de um lote de cada um dos materiais foram pré-condicionadas, por 18 horas, entre folhas de papel umedecido, a $25{ }^{\circ} \mathrm{C}$. Duas metodologias destinadas ao preparo das sementes foram testadas: corte lateral e corte lateral seguido de imersão das sementes em água, a $30{ }^{\circ} \mathrm{C}$, durante uma hora, e posterior remoção do tegumento. Para a coloração, 100 sementes de cada um dos lotes foram imersas em solução de tetrazólio nas concentrações de $0,075 \%, 0,15 \%$ e $1 \%$, no interior de recipientes plásticos de $50 \mathrm{~mL}$, por duas e quatro horas, a $35{ }^{\circ} \mathrm{C}$, no escuro. Ao término do período de coloração, as sementes foram lavadas em água corrente, seccionadas longitudinalmente através do centro do eixo embrionário e avaliadas apenas quanto à uniformidade e à intensidade da coloração apresentadas pelos tecidos, sendo classificadas em: não coloridas, parcialmente coloridas, coloridas com intensidades fraca, adequada ou forte.

Para avaliar a eficiência do teste, cinco lotes de sementes da cv. 'Cunningham' e cinco lotes de sementes do híbrido interespecífico (L. leucocephala x L. diversifolia) CPAC 4111 foram submetidos aos testes de germinação, emergência de plântulas e tetrazólio.

$\mathrm{O}$ teste de germinação foi conduzido em rolos de papel, a $25{ }^{\circ} \mathrm{C}$, com avaliações aos quatro e dez dias, conforme as Regras para Análise de Sementes (Brasil, 1992). Foram empregadas 200 sementes de cada lote, em quatro subamostras de 50 sementes. Ao final do teste, determinou-se a percentagem de plântulas normais.

Para o teste de emergência de plântulas, 200 sementes de cada lote, em quatro subamostras de 50 , foram semeadas em bandejas plásticas de 3,6 L, empregando areia de textura média como substrato, e cobertas por uma camada de dois centímetros de areia. As bandejas foram mantidas em casa de vegetação e irrigadas diariamente. A avaliação foi realizada aos 15 dias após a semeadura, determinando-se a percentagem de plântulas emergidas.

Quanto ao teste de tetrazólio, dentre as metodologias testadas para o preparo e coloração das sementes, empregouse aquela que proporcionou coloração uniforme e de intensidade adequada para avaliação. Para isso, 100 sementes de cada lote, em quatro subamostras de 25 sementes, foram pré-condicionadas por 18 horas, entre papel umedecido, a 25 ${ }^{\circ} \mathrm{C}$, sendo posteriormente submetidas ao corte lateral, seguido da imersão em água, a $30{ }^{\circ} \mathrm{C}$, por uma hora, para remoção do tegumento. Para a coloração, o teste foi conduzido com solução de tetrazólio a $0,15 \%$, por duas horas, a $35{ }^{\circ} \mathrm{C}$. Após o período de coloração, as sementes foram lavadas em água corrente, seccionadas longitudinalmente através do centro do eixo embrionário e analisadas sob microscópio estereoscópico de quatro aumentos. A interpretação baseouse na localização e intensidade de coloração dos tecidos embrionários, presença e localização de danos, e as sementes foram classificadas em viáveis e não viáveis. Foram classificadas em viáveis as sementes com coloração rosa claro brilhante, sem danos, ou com lesões superficiais em áreas não críticas dos cotilédones, ou seja, distantes do ponto de ligação destes com o eixo embrionário, ocupando menos de $50 \%$ de sua área total. Como não viáveis, foram classificadas as sementes que apresentavam coloração rosa intenso, branca ou com aspecto de mosaico na região de ligação dos cotilédones com o eixo-embrionário ou ocupando área superior a $50 \%$ do seu total. Também foram consideradas não viáveis as sementes que apresentaram radícula completamente descolorida ou coloração rosa intenso atingindo o cilindro central ou mais da metade da extensão da radícula. Os resultados foram expressos em percentagem de sementes viáveis.

Os experimentos foram realizados em delineamento inteiramente casualizado, com quatro repetições. Os valores observados nos testes de germinação, emergência e tetrazólio foram transformados em arc sen e, as médias, comparadas pelo teste de Tukey $(\mathrm{p}<0,05)$. Adicionalmente, calcularamse os coeficientes de correlação simples de Pearson (r) entre as médias dos valores obtidos nos testes de germinação, emergência de plântulas e tetrazólio. A significância dos valores de $r$ foi determinada pelo teste $t(p<0,01)$.

\section{RESULTADOS E DISCUSSÃO}

Verificou-se que as sementes submetidas ao corte lateral, sem remoção do tegumento, não se coloriram adequadamente, independente da concentração da solução de tetrazólio e do período de coloração (Tabela 1). Em geral, a solução de tetrazólio não penetrou uniformemente nos tecidos das sementes, concentrando-se na região do corte, razão da elevada percentagem de sementes classificadas em parcialmente coloridas (Figura 1).

Por outro lado, sementes submetidas ao corte lateral, seguido da imersão em água e posterior remoção do tegumento, apresentaram padrões variados de coloração, dependendo da combinação entre a concentração da solução de tetrazólio e período de coloração (Tabela 2). Muitas sementes de espécies da família Fabaceae apresentam o tegumento impermeável à 
água, o que dificulta a penetração da solução de tetrazólio durante o teste e determinam a necessidade de sua remoção para que a coloração das sementes ocorra uniformemente, conforme observado em sementes de farinha-seca (Zucareli et al., 2001), guapuruvu (Ferreira et al., 2007), coração-de-negro (Pinto et al., 2008) e amendoim (Bittencourt e Vieira, 1999).

TABELA 1. Padrão de coloração de sementes de leucena submetidas ao corte lateral e imersão em soluções de tetrazólio a $0,075 \%, 0,15 \%$ e $1 \%$, por duas e quatro horas.

\begin{tabular}{|c|c|c|c|c|c|c|c|}
\hline \multirow[b]{3}{*}{ Materiais } & \multirow{3}{*}{$\begin{array}{l}\text { Tempo de } \\
\text { coloração }\end{array}$} & \multirow{3}{*}{$\begin{array}{c}\text { Solução } \\
\text { de } \\
\text { Tetrazólio }\end{array}$} & \multicolumn{5}{|c|}{ Padrão de coloração (\%) } \\
\hline & & & \multirow[b]{2}{*}{$\begin{array}{c}\text { Não } \\
\text { coloridas }\end{array}$} & \multirow[b]{2}{*}{$\begin{array}{c}\text { Parcialmente } \\
\text { coloridas }\end{array}$} & \multicolumn{3}{|c|}{ Coloridas } \\
\hline & & & & & Fraca & adequada & Forte \\
\hline \multirow{6}{*}{$\begin{array}{c}\text { cv. } \\
\text { 'Cunningham' }\end{array}$} & \multirow{3}{*}{$2 \mathrm{~h}$} & $0,075 \%$ & 4 & 96 & 0 & 0 & 0 \\
\hline & & $0,15 \%$ & 9 & 90 & 1 & 0 & 0 \\
\hline & & $1 \%$ & 2 & 87 & 2 & 2 & 7 \\
\hline & \multirow{3}{*}{$4 \mathrm{~h}$} & $0,075 \%$ & 4 & 96 & 0 & 0 & 0 \\
\hline & & $0,15 \%$ & 0 & 100 & 0 & 0 & 0 \\
\hline & & $1 \%$ & 1 & 94 & 0 & 5 & 0 \\
\hline \multirow{6}{*}{$\begin{array}{l}\text { híbrido CPAC } \\
4111\end{array}$} & \multirow{3}{*}{$2 \mathrm{~h}$} & $0,075 \%$ & 11 & 89 & 0 & 0 & 0 \\
\hline & & $0,15 \%$ & 5 & 95 & 0 & 0 & 0 \\
\hline & & $1 \%$ & 7 & 83 & 3 & 1 & 6 \\
\hline & \multirow{3}{*}{$4 \mathrm{~h}$} & $0,075 \%$ & 16 & 84 & 0 & 0 & 0 \\
\hline & & $0,15 \%$ & 3 & 95 & 2 & 0 & 0 \\
\hline & & $1 \%$ & 3 & 86 & 2 & 2 & 7 \\
\hline
\end{tabular}

TABELA 2. Padrão de coloração de sementes de leucena submetidas ao corte lateral e remoção do tegumento e imersão em soluções de tetrazólio a $0,075 \%, 0,15 \%$ e $1 \%$, por duas e quatro horas.

\begin{tabular}{|c|c|c|c|c|c|c|c|}
\hline \multirow[b]{3}{*}{ Materiais } & \multirow{3}{*}{$\begin{array}{l}\text { Tempo de } \\
\text { coloração }\end{array}$} & \multirow{3}{*}{$\begin{array}{l}\text { Solução } \\
\text { de } \\
\text { Tetrazólio }\end{array}$} & \multicolumn{5}{|c|}{ Padrão de coloração (\%) } \\
\hline & & & \multirow[b]{2}{*}{$\begin{array}{c}\text { Não } \\
\text { coloridas }\end{array}$} & \multirow[b]{2}{*}{$\begin{array}{c}\text { Parcialmente } \\
\text { coloridas }\end{array}$} & \multicolumn{3}{|c|}{ Coloridas } \\
\hline & & & & & Fraca & adequada & Forte \\
\hline \multirow{6}{*}{$\begin{array}{c}\text { cv. } \\
\text { 'Cunningham' }\end{array}$} & \multirow{3}{*}{$2 \mathrm{~h}$} & $0,075 \%$ & 1 & 0 & 92 & 7 & 0 \\
\hline & & $0,15 \%$ & 2 & 0 & 0 & 95 & 3 \\
\hline & & $1 \%$ & 3 & 2 & 0 & 0 & 95 \\
\hline & \multirow{3}{*}{$4 \mathrm{~h}$} & $0,075 \%$ & 3 & 0 & 30 & 67 & 0 \\
\hline & & $0,15 \%$ & 0 & 0 & 0 & 99 & 1 \\
\hline & & $1 \%$ & 0 & 0 & 0 & 0 & 100 \\
\hline \multirow{6}{*}{$\begin{array}{c}\text { híbrido CPAC } \\
4111\end{array}$} & \multirow{3}{*}{$2 \mathrm{~h}$} & $0,075 \%$ & 9 & 2 & 79 & 0 & 10 \\
\hline & & $0,15 \%$ & 7 & 0 & 2 & 79 & 12 \\
\hline & & $1 \%$ & 4 & 0 & 0 & 0 & 96 \\
\hline & \multirow{3}{*}{$4 \mathrm{~h}$} & $0,075 \%$ & 1 & 0 & 25 & 65 & 9 \\
\hline & & $0,15 \%$ & 2 & 0 & 4 & 79 & 15 \\
\hline & & $1 \%$ & 8 & 0 & 3 & 0 & 89 \\
\hline
\end{tabular}




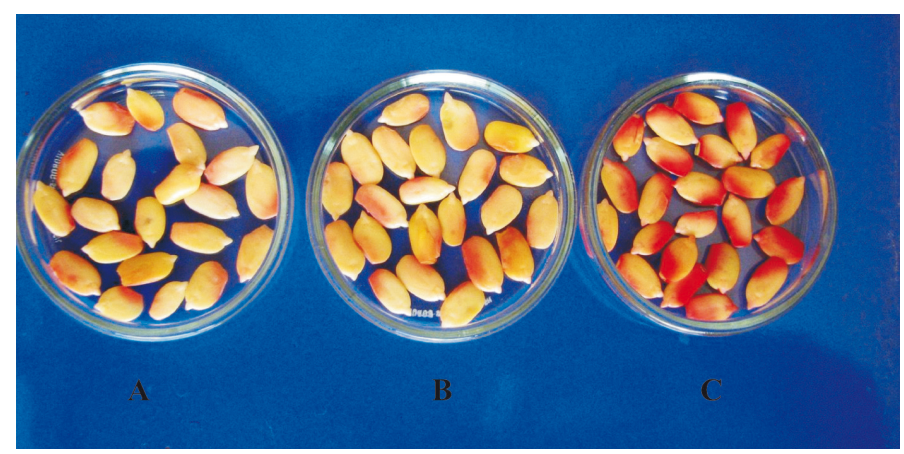

FIGURA 1. Sementes de leucena submetidas ao corte lateral e quatro horas de coloração em soluções de tetrazólio a $0,075 \%$ (A), $0,15 \%$ (B) e $1 \%$ (C).

No presente trabalho, o pré-condicionamento, seguido de corte lateral e imersão das sementes em água, a $30^{\circ} \mathrm{C}$, por uma hora, facilitou a remoção do tegumento. De forma semelhante, a imersão direta das sementes em água a $40{ }^{\circ} \mathrm{C}$ por 30 minutos é prática recomendável para facilitar a remoção do tegumento de sementes de abobrinha a serem submetidas ao teste de tetrazólio (Barros et al., 2005).

Para a coloração das sementes, a combinação entre concentração da solução de tetrazólio e período que permitiu a obtenção dos melhores resultados, em termos de intensidade e uniformidade, foi a concentração da solução de tetrazólio a $0,15 \%$ e o período de coloração de duas horas, tanto para a cultivar quanto para o híbrido. Sementes submetidas à solução de tetrazólio a $0,075 \%$, por quatro horas, apresentaram intensidade razoável de coloração, porém menos uniforme, comparativamente à solução de tetrazólio a $0,15 \%$, por duas e quatro horas. A solução de tetrazólio a $1 \%$ promoveu coloração muito intensa das sementes, dificultando a interpretação do teste (Figura 2). Esses resultados contradizem as recomendações das Regras para Análise de Sementes (Brasil, 1992), que indicam concentrações de 0,5 a 1,0\% da solução de tetrazólio e períodos de coloração de 6 a 24 horas. É provável que a remoção do tegumento das sementes tenha favorecido a absorção mais rápida da solução de tetrazólio pelos tecidos, reduzindo o período de exposição das sementes à solução, mesmo empregando concentrações inferiores às recomendadas.

$\mathrm{Na}$ Tabela 3, são apresentados os resultados obtidos nos testes de germinação, emergência de plântulas e tetrazólio relativos à viabilidade das sementes de leucena. Observou-se correlação entre os resultados dos testes quanto à separação dos lotes em níveis de qualidade fisiológica. Para as sementes do híbrido CPAC 4111, o teste de tetrazólio permitiu separar os lotes de qualidade fisiológica superior (lotes 4 e 5) dos lotes de qualidade inferior (lotes 1, 2 e 3 ) da mesma forma que os testes de germinação e emergência de plântulas. Em relação às sementes da cv. 'Cunningham', o teste de tetrazólio permitiu separar os lotes de qualidade superior (lotes 3,4 e 5) dos lotes de qualidade inferior (lotes 1 e 2), semelhante aos resultados obtidos no teste de emergência de plântulas. No caso das sementes da cv. 'Cunningham', pelos resultados do teste de germinação houve maior separação entre os lotes, evidenciando o lote 5 como de qualidade fisiológica superior, os lotes 3 e 4 como de qualidade intermediária e os lotes 1 e 2 como de qualidade inferior. Todavia, comparando os resultados dos diferentes testes em relação à estimativa da viabilidade das sementes, para cada lote, verificou-se que não ocorreram diferenças significativas entre eles, à exceção dos resultados obtidos para o lote 4 do híbrido CPAC 4111, cujos resultados relativos à emergência de plântulas foram superiores aos obtidos nos testes de germinação e tetrazólio. Esses resultados confirmam a possibilidade de utilização do teste de tetrazólio para avaliar a viabilidade de sementes de leucena, conforme já observado em diversas espécies, como braquiária (Novembre et al., 2006), soja (Costa et al., 1998), milho (Chamma e Novembre, 2007), amendoim (Carvalho et al., 2009), algodão (Cervi e Mendonça, 2009), entre outras.

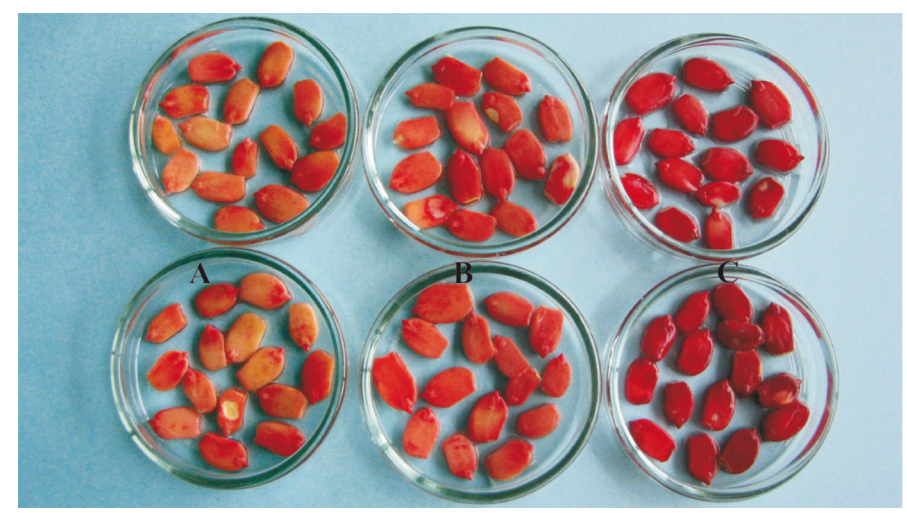

FIGURA 2. Sementes de leucena submetidas ao corte lateral e posterior remoção do tegumento, após duas (acima) e quatro (abaixo) horas de coloração em soluções de tetrazólio a $\mathbf{0 , 0 7 5 \%}$ (A), $0,15 \%$ (B) e $1 \%$ (C). 
TABELA 3. Viabilidade de sementes de leucena da cultivar 'Cunningham' e do híbrido interespecífico ( $L$. leucocephala $\mathrm{x}$ L. diversifolia) CPAC 4111, avaliadas pelos testes de germinação (G), emergência de plântulas (E) e tetrazólio (TZ).

\begin{tabular}{|c|c|c|c|c|c|c|c|c|c|c|c|c|}
\hline \multirow{3}{*}{ Testes } & \multicolumn{12}{|c|}{ Viabilidade (\%) } \\
\hline & \multicolumn{6}{|c|}{ Lotes (cv. 'Cunningham') } & \multicolumn{6}{|c|}{ Lotes (híbrido CPAC 4111) } \\
\hline & 1 & 2 & 3 & 4 & 5 & CV $(\%)$ & 1 & 2 & 3 & 4 & 5 & CV $(\%)$ \\
\hline $\mathrm{G}$ & $61 \mathrm{Ad}$ & $83 \mathrm{Ac}$ & $93 \mathrm{Ab}$ & $94 \mathrm{Ab}$ & $100 \mathrm{Aa}$ & 3,73 & $58 \mathrm{Ac}$ & $64 \mathrm{Abc}$ & $73 \mathrm{Ab}$ & $85 \mathrm{Ba}$ & $93 \mathrm{Aa}$ & 5,82 \\
\hline $\mathrm{E}$ & $65 \mathrm{Ac}$ & $84 \mathrm{Abc}$ & $98 \mathrm{Aa}$ & $96 \mathrm{Aab}$ & $97 \mathrm{Aab}$ & 8,87 & $64 \mathrm{Ab}$ & $63 \mathrm{Ab}$ & $73 \mathrm{Ab}$ & $97 \mathrm{Aa}$ & $95 \mathrm{Aa}$ & 7,25 \\
\hline $\mathrm{TZ}$ & $67 \mathrm{Ab}$ & $83 \mathrm{Ab}$ & 98Aa & $97 \mathrm{Aa}$ & $96 \mathrm{Aa}$ & 7,94 & $55 \mathrm{Ac}$ & $71 \mathrm{Ab}$ & $72 \mathrm{Ab}$ & $87 \mathrm{Ba}$ & $88 \mathrm{Aa}$ & 5,10 \\
\hline $\mathrm{CV}(\%)$ & 5,19 & 5,28 & 8,04 & 9,02 & 6,01 & & 5,47 & 4,67 & 6,07 & 7,13 & 6,00 & \\
\hline
\end{tabular}

Médias seguidas pela mesma letra, maiúscula nas colunas e, em cada genótipo, minúscula nas linhas, não diferem entre si pelo teste de Tukey (p<0,05).

Os valores dos coeficientes de correlação entre os resultados dos testes de germinação, emergência de plântulas e tetrazólio (Tabela 4) evidenciaram alta correlação: tetrazólio e germinação $(r=0,96)$ e tetrazólio e emergência de plântulas $(r=0,93)$. A correlação positiva e significativa entre esses testes também foi observada em sementes de soja (Barros e Marcos Filho, 1997), abobrinha (Barros et al., 2005) e melancia (Bhering et al., 2005; Nery et al., 2007).

TABELA 4. Coeficientes de correlação simples de Pearson entre os resultados dos testes de germinação, emergência de plântulas e tetrazólio em sementes de leucena.

\begin{tabular}{lccc}
\hline Testes & Germinação & Emergência & Tetrazólio \\
\hline Germinação & 1,00 & $0,96^{* *}$ & $0,96^{* *}$ \\
Emergência & & 1,00 & $0,93^{* *}$ \\
Tetrazólio & & & 1,00 \\
\hline
\end{tabular}

**Significativo ao nivel de $1 \%$ de probabilidade pelo teste $\mathrm{t}$.

\section{CONCLUSÕES}

O teste de tetrazólio é eficiente para avaliar a viabilidade de sementes de leucena;

Após o pré-condicionamento, por 18 horas a $25^{\circ} \mathrm{C}$ ,recomenda-se o corte lateral das sementes, seguido da imersão em água, a $30^{\circ} \mathrm{C}$, por uma hora, e posterior remoção do tegumento;
Para a coloração, deve-se utilizar solução de tetrazólio a $0,15 \%$, por duas horas, a $35^{\circ} \mathrm{C}$.

\section{AGRADECIMENTOS}

À Unipasto - Associação para o Fomento à Pesquisa de Melhoramento de Forrageiras Tropicais, pelo apoio financeiro à execução dos experimentos.

\section{REFERÊNCIAS}

BARROS, A.S.R.; MARCOS FILHO, J. Testes para avaliação rápida do vigor de sementes de soja. Revista Brasileira de Sementes, v.19, n.2, p.288-294, 1997.

BARROS, D.I.; DIAS, D.C.F.S.; BHERING, M.C.; DIAS, L.A.S.; ARAÚJO, E.F. Uso do teste de tetrazólio para avaliação da qualidade fisiológica de sementes de abobrinha. Revista Brasileira de Sementes, v.27, n.2, p.165-171, 2005.

BHERING, M.C.; DIAS, D.C.F.S.; BARROS, D.I.Adequação da metodologia do teste de tetrazólio para avaliação da qualidade fisiológica de sementes de melancia. Revista Brasileira de Sementes, v.27, n.1, p.176-182, 2005.

BITTENCOURT, S.R.M.; VIEIRA, R.D. Metodologia do teste de tetrazólio em amendoim. In: KRZYZANOWSKI, F.C.; VIEIRA, R.D.; FRANÇA NETO, J.B. (Ed.). (Vigor de sementes: conceitos e testes. Londrina: ABRATES, 1999. cap. 8.2, p.1-8.

BRASIL. Ministério da Agricultura e Reforma Agrária. Secretaria Nacional de Defesa Agropecuária. Departamento Nacional de Defesa Vegetal. Coordenação de Laboratório Vegetal. Regras para análise de sementes. Brasília, DF, 
1992. 365p.

CARVALHO, N.M.; SILVA, J.B.; SILVEIRA, C.M.; HORVAT, R.A. Método alternativo para submeter sementes de amendoim à solução de tetrazólio. Revista Brasileira de Sementes, v.31, n.1, p.18-22, 2009.

CERVI, F.; MENDONÇA, E.A.F. Adequação do teste de tetrazólio para sementes de algodoeiro. Revista Brasileira de Sementes, v.31, n.1, p.177-186, 2009.

CHAMMA, H.M.C.P.; NOVEMBRE, A.D.L.C. Teste de tetrazólio para as sementes de milho: períodos de hidratação e de coloração das sementes. Revista Brasileira de Sementes, v.29, n.2, p.125-129, 2007.

COSTA, N.P.; FRANÇA NETO, J.B.; KRZYZANOWSKI, F.C.; HENNING, A.A.; OLIVEIRA, M.C.N. Procedimento alternativo no teste de tetrazólio em sementes de soja. Pesquisa Agropecuária Brasileira, v.33, n.6, p.869-877, 1998.

DELOUCHE, J.C.; STILL, T.W.; RASPET, M.; LIENHARD, M. O teste de tetrazólio para viabilidade da semente. Brasília, DF: AGIPLAN, 1976. 103p.

FERREIRA, R.A.; OLIVEIRA, L.M.; TONETTI, O.A.O.; DAVIDE, A.C. Comparação da viabilidade de sementes de Schizolobium parahyba (Vell.) Blake - Leguminosae Caesalpinioideae, pelos testes de germinação e tetrazólio. Revista Brasileira de Sementes, v.29, n.3, p.73-79, 2007.

FRANÇA NETO, J.B.; KRZYZANOWSKI, F.C.; COSTA, N.P. O teste de tetrazólio em sementes de soja. Londrina: Embrapa CNPSo, 1998. 72p. (EMBRAPA-CNPSo. Documentos, 116).

FRANÇA NETO, J.B. Testes de tetrazólio para determinação do vigor de sementes. In: KRZYZANOWSKI, F.C.; VIEIRA, R.D.; FRANÇA NETO, J.B. (Ed.). Vigor de sementes: conceitos e testes. Londrina: ABRATES, 1999. cap. 8, p.1-7.

MANELLA, M.Q.; LOURENÇO, A.J.; LEME, P.R. Recria de bovinos nelore em pastos de Brachiaria brizantha com suplementação protéica ou com acesso a banco de proteína de Leucaena leucocephala. Desempenho animal. Revista Brasileira de Zootecnia, v.31, n.6, p.2274-2282, 2002.

NERY, M.C.; CARVALHO, M.L.M.; OLIVEIRA, L.M. Teste de tetrazólio para avaliação da qualidade fisiológica de sementes de melancia. Semina, v.28, n.3, p.365-372, 2007.

NOVEMBRE, A.D.L.C.; CHAMMA, H.M.C.P.; GOMES, R.B.R. Viabilidade das sementes de braquiária pelo teste de tetrazólio. Revista Brasileira de Sementes, v.28, n.2, p.147151, 2006.

PINTO, T.L.F.; BRANCALION, P.H.S.; NOVEMBRE, A.D.L.C.; CICERO, S.M. Avaliação da viabilidade de sementes de coração-de-negro (Poecilanthe parviflora Benth. - Fabaceae - Faboideae) pelo teste de tetrazólio. Revista Brasileira de Sementes, v.30, n.1, p.208-214, 2008.

SOUZA, A.A.; ESPÍNDOLA, G.B. Bancos de proteína de leucena e de guandu para suplementação de ovinos mantidos em pastagens de capim-buffel. Revista Brasileira de Zootecnia, v.29, n.2, p.365-372, 2000.

ZUCARELI, C.; MALAVASI, M.M.; FOGAÇA, C.A.; MALAVASI, U.C. Preparo e coloração de sementes de farinha-seca (Albizia hasslerii (Chodat) Burr.) para o teste de tetrazólio. Revista Brasileira de Sementes, v.23, n.2, p.186191, 2001. 